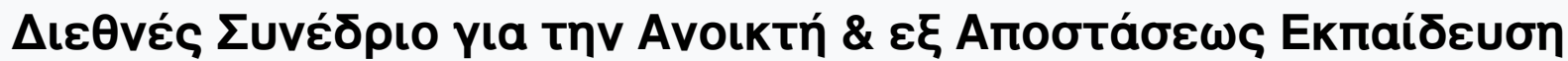

Tón. 6, Ap. 1A (2011)

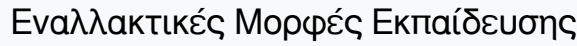

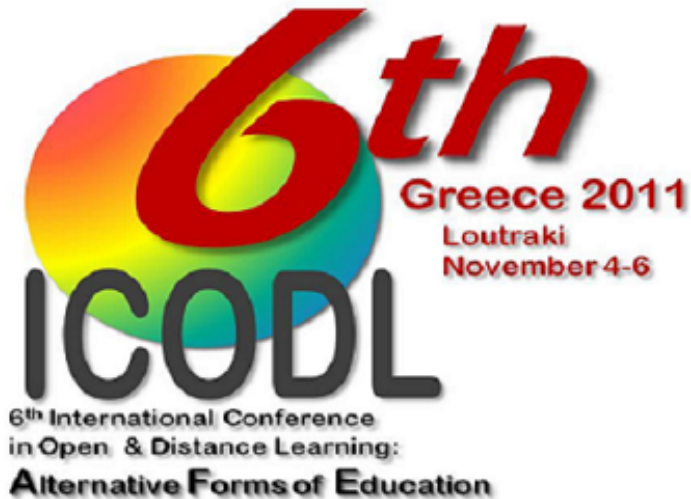

TOMOEA

PART/MEPOEA

Alternative Forms of Education

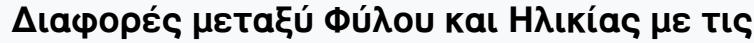

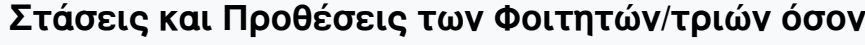

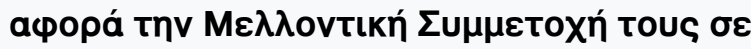

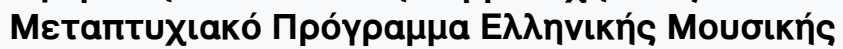

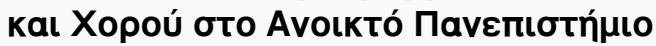

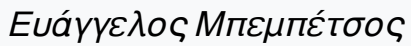

doi: $\underline{10.12681 / \text { icodl. } 688}$ 


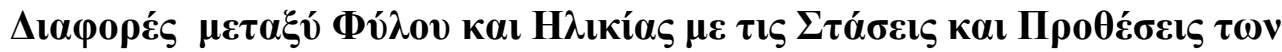

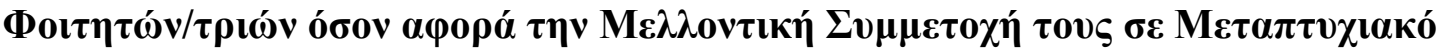

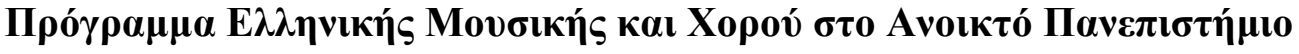

\title{
Differences between Gender, Age, Attitudes and Intentions among University Students on their Future Student Postgraduate Greek Music and Dance Participation within the Hellenic Open University
}

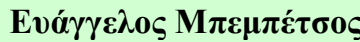

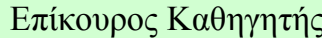

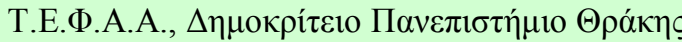 \\ empempet@phyed.duth.gr
}

\section{Пврі́ $\eta \psi \eta$}

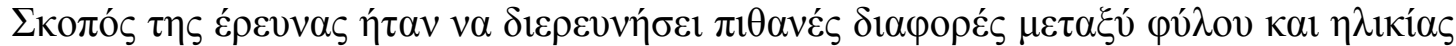

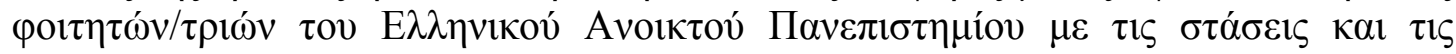

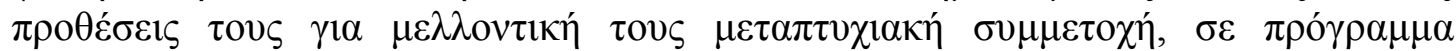

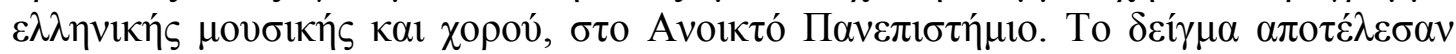

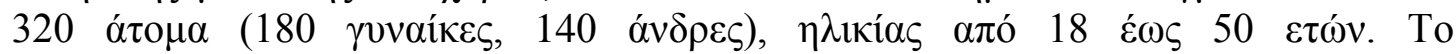

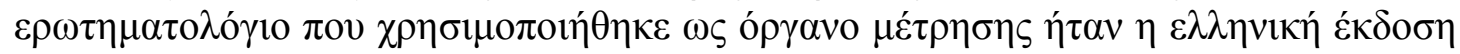

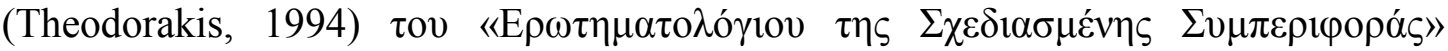

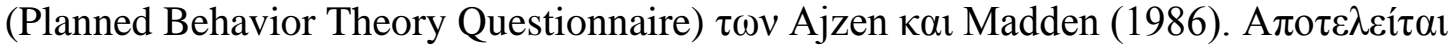

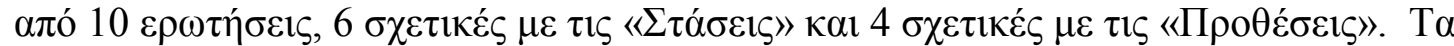

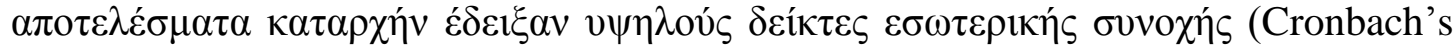

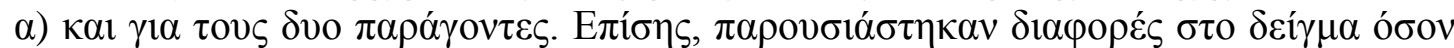

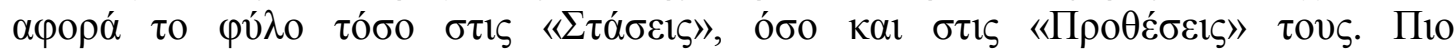

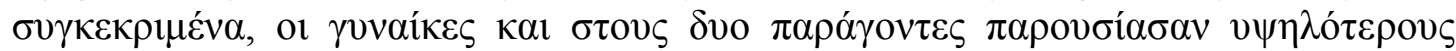

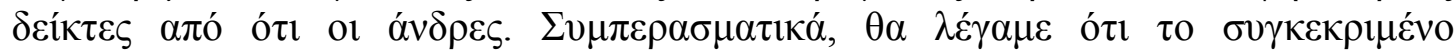

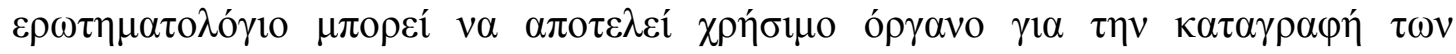

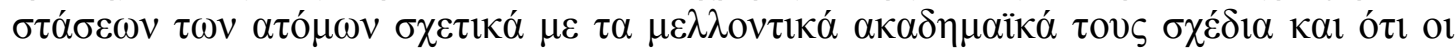

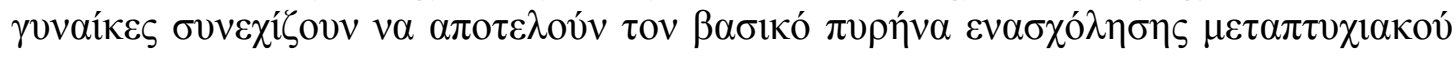

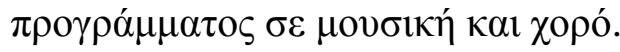

\begin{abstract}
The aim of the study was to investigate any possible relations between gender, age, attitudes and intention among Greek University students, on their future postgraduate Greek music and dance participation, within the University. The sample consisted of 320 students ( 210 female, 110 male) between the ages of 18 up to 50. The instrument that was used was the Greek version (Theodorakis, 1994) of the "Planned Behavior Theory Questionnaire" (Ajzen \& Madden, 1986). It included 10 items, 6 for the variable of "Attitudes" and 4 for the variable of "Intentions". The results revealed high internal consistency among them. Also, gender differences were noticed, were women had higher scores than men on both variables. Overall, the study indicated the usefulness of the specific instrument on the investigation of students' future attitudes and intentions and that women are the major "component" of a post graduate program on music and dance.
\end{abstract}




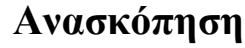

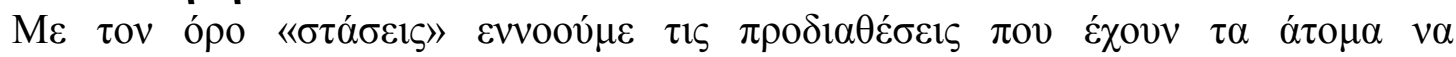

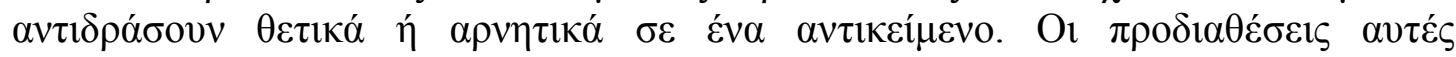

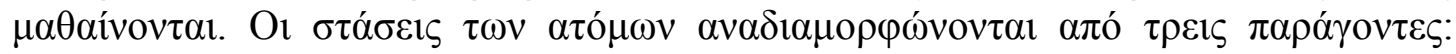

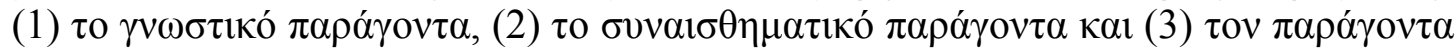

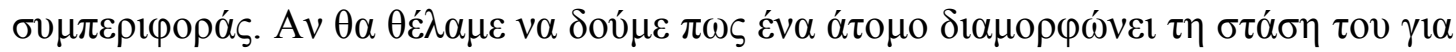

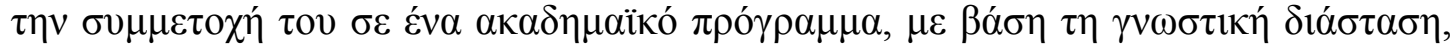

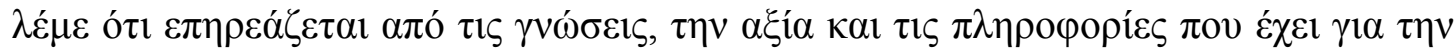

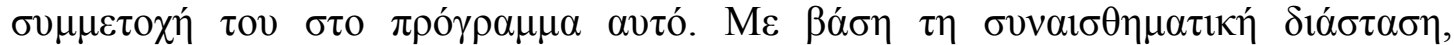

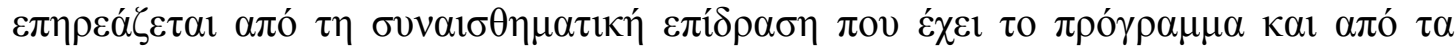

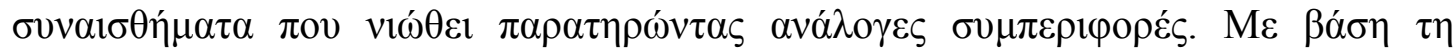

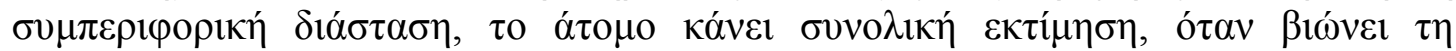

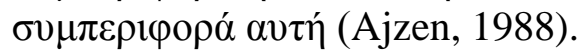

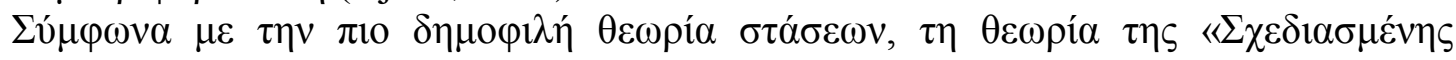

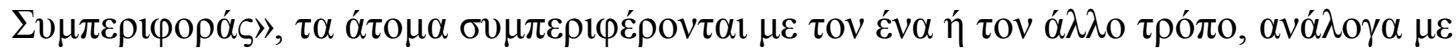

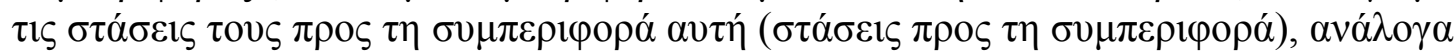

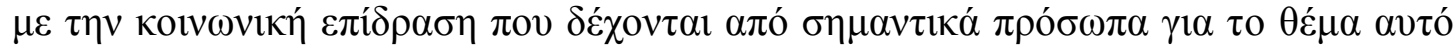

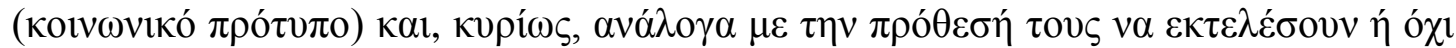

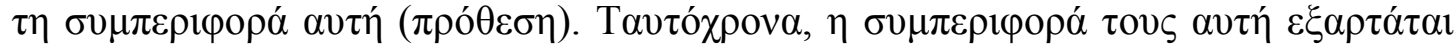

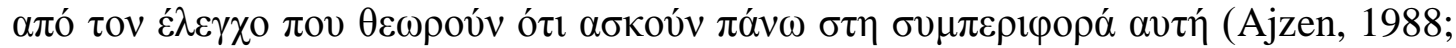

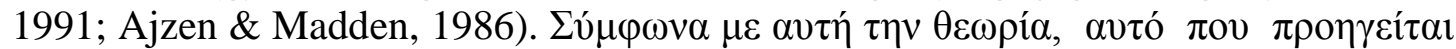

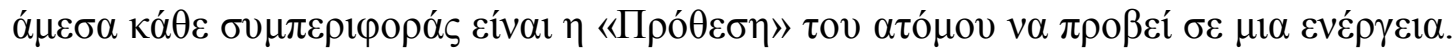

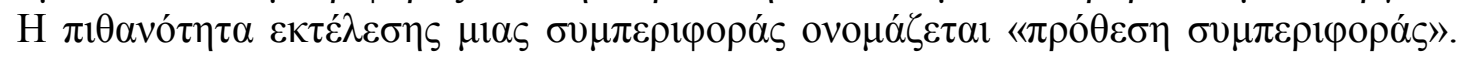

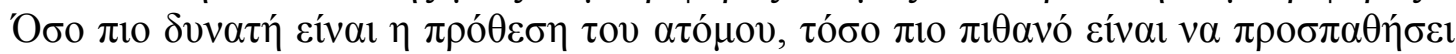

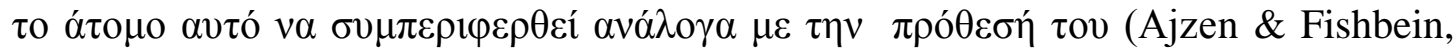

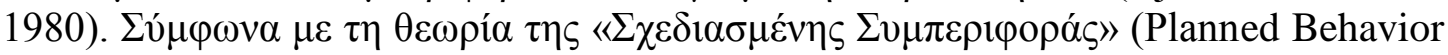

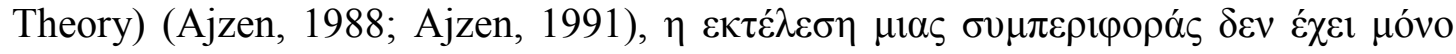

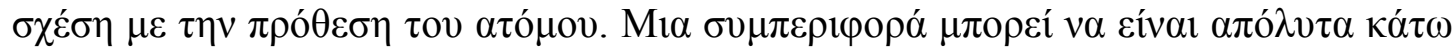

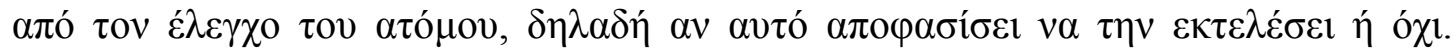

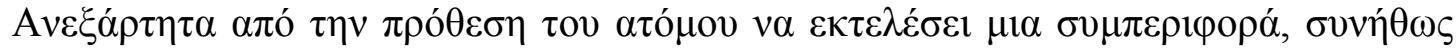

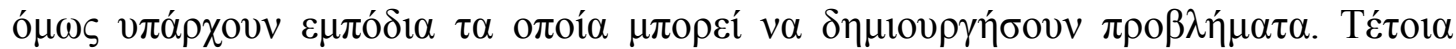

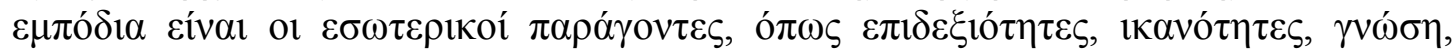

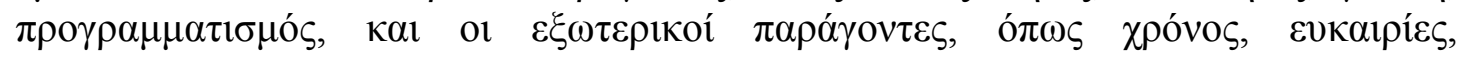

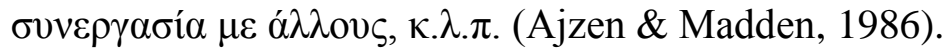

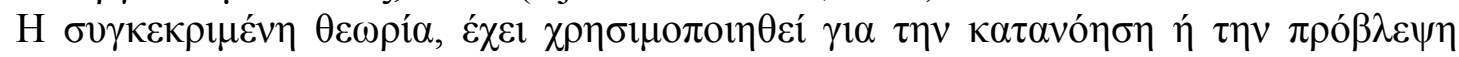

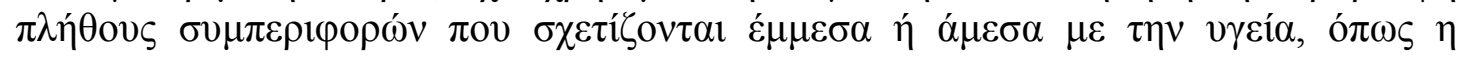

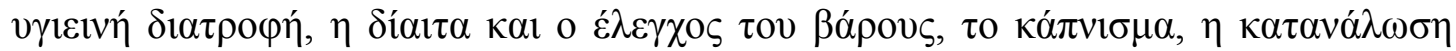

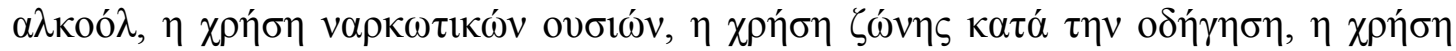

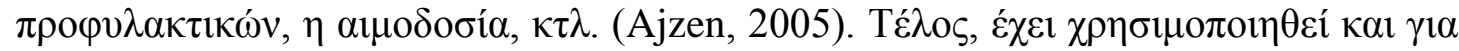

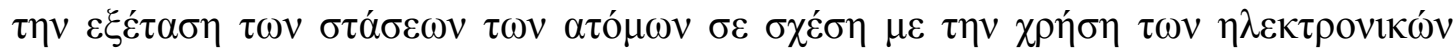

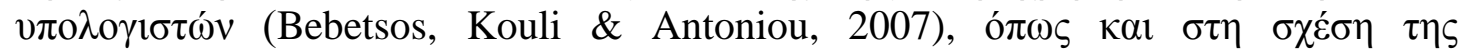
$\varepsilon v \alpha \sigma \chi \delta ́ \lambda \eta \sigma \eta ́ \varsigma \mu$ б

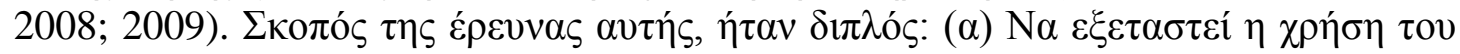

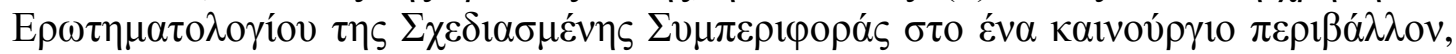

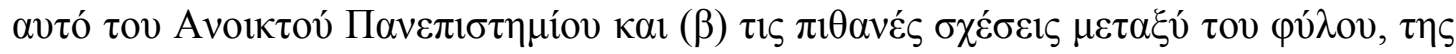

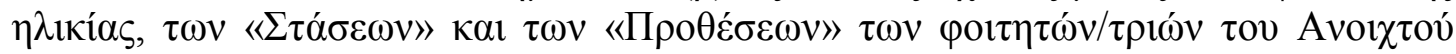

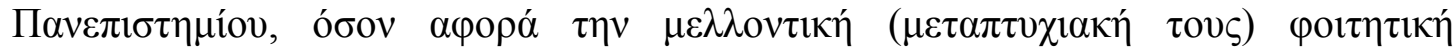

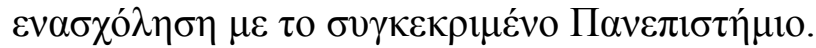




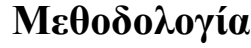

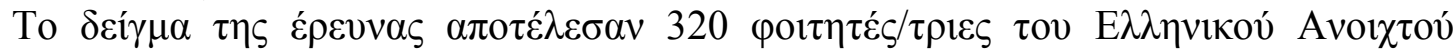

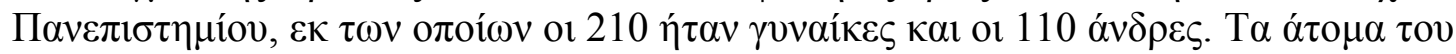

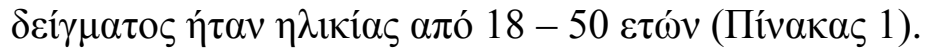

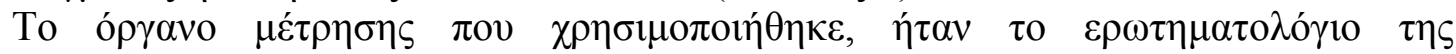

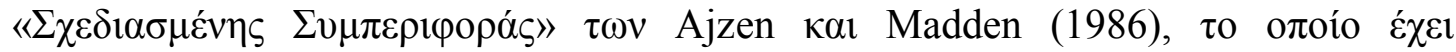

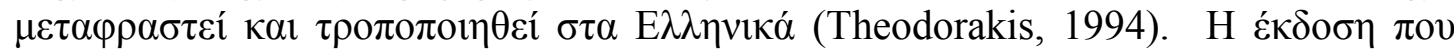

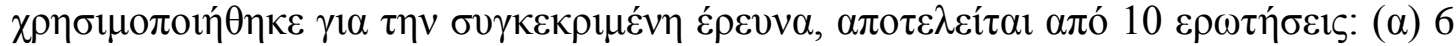

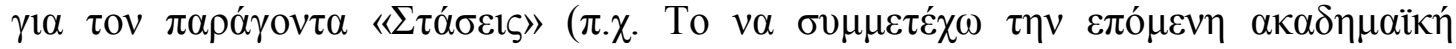

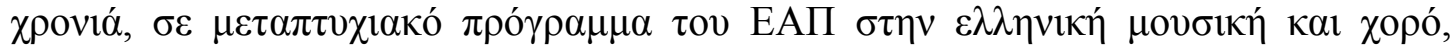

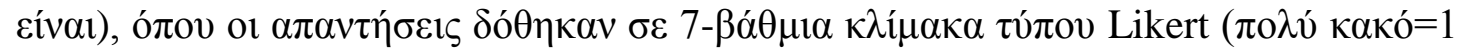

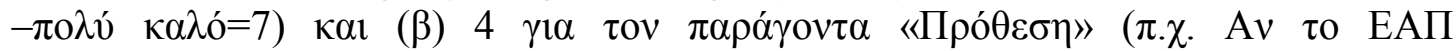

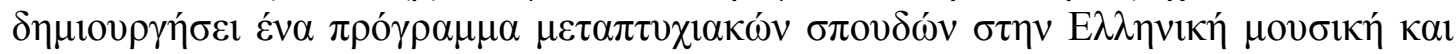

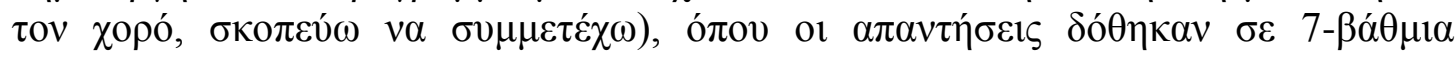

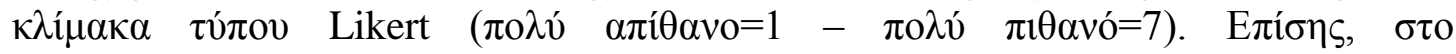

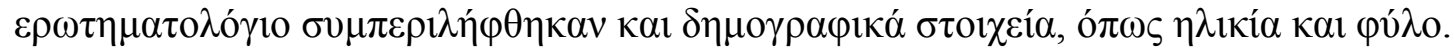

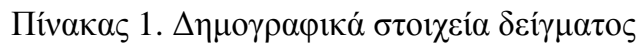

\begin{tabular}{|c|c|c|}
\hline $\begin{array}{c}\mathrm{AN} \Delta \mathrm{PA} \Sigma \\
140\end{array}$ & \multicolumn{2}{|c|}{$\begin{array}{c}\Gamma \text { YNAIKA } \\
180 \\
\end{array}$} \\
\hline \multicolumn{3}{|c|}{ H $\Lambda \mathrm{IKIA}$} \\
\hline $18-30$ & $31-40$ & $41-50$ \\
\hline $57(18.7 \%)$ & $147(48.2 \%)$ & $101(33.1 \%)$ \\
\hline
\end{tabular}

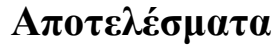

\section{Avód}

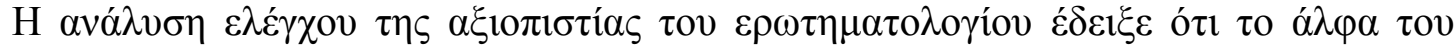

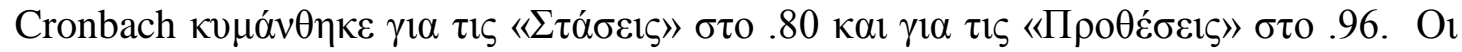

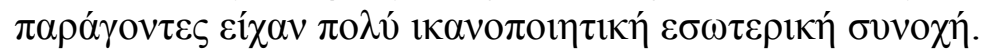

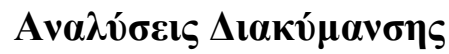

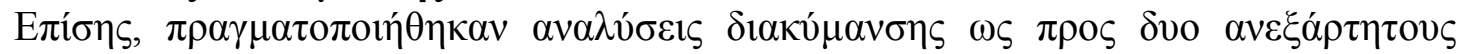

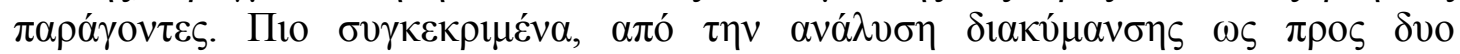

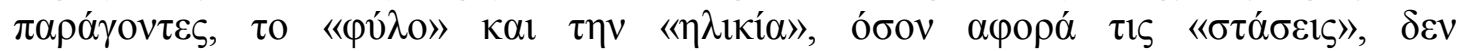

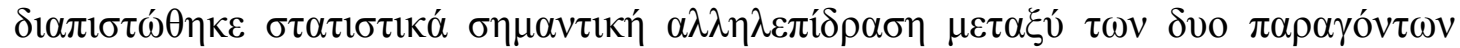

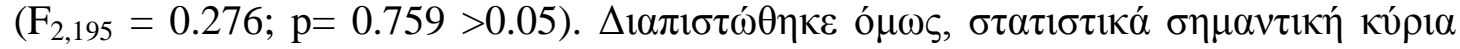

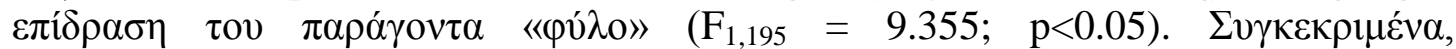

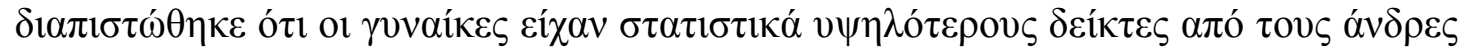
(Пívaкas 2).

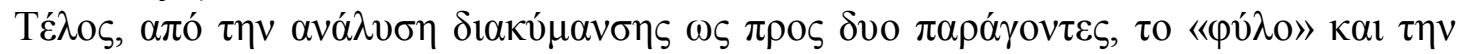

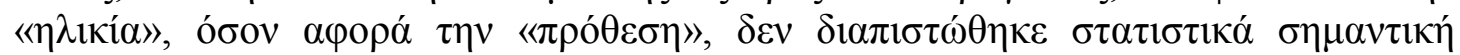

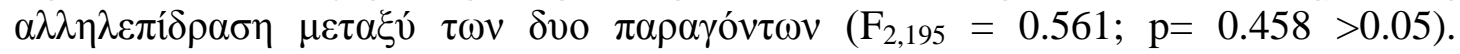

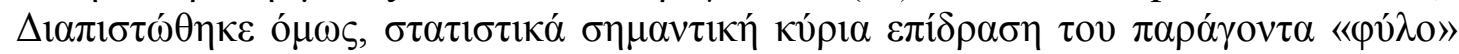

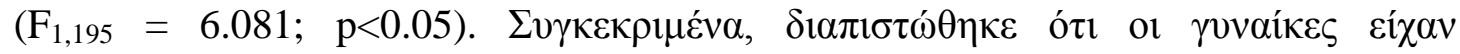

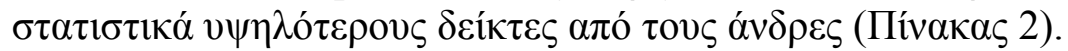




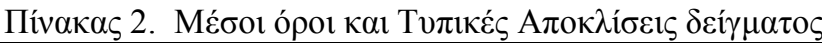

\begin{tabular}{|c|c|c|c|c|}
\hline \multirow[t]{2}{*}{ ПАРАГONTЕ $\Sigma$} & \multicolumn{2}{|c|}{ ГYNAIKE $\Sigma$} & \multicolumn{2}{|c|}{$\mathrm{AN} \triangle \mathrm{PE} \Sigma$} \\
\hline & $\mathrm{M}$ & TA & $\mathrm{M}$ & TA \\
\hline$\Sigma \tau \alpha ́ \sigma \varepsilon 1 \zeta$ & 5.812 & 1.50 & 4.993 & 2.05 \\
\hline Про́$\theta \varepsilon \sigma \eta$ & 4.302 & 2.87 & 3.86 & 3.18 \\
\hline
\end{tabular}

\section{$\Sigma v \zeta \dot{\eta} \tau \eta \sigma \eta$}

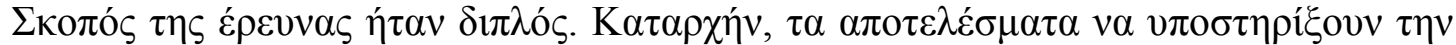

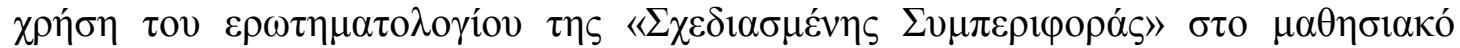

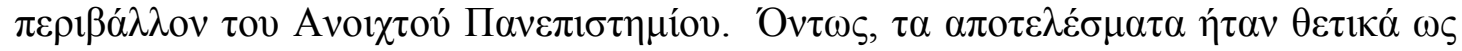

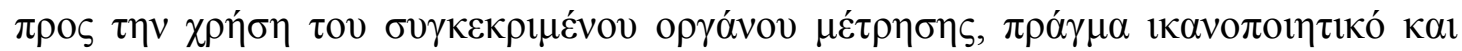

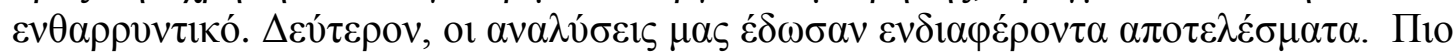

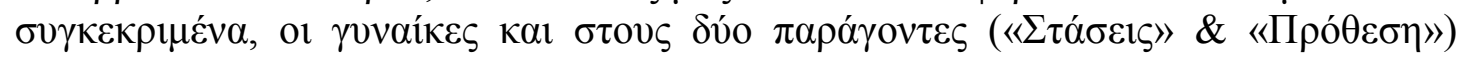

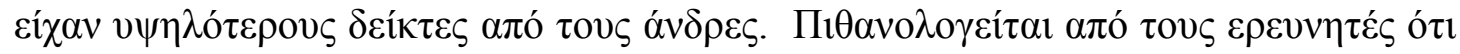

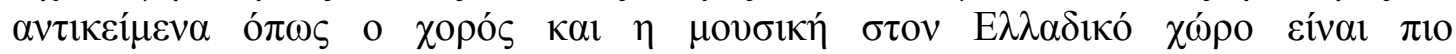

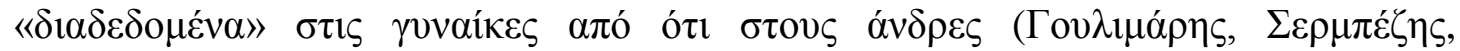

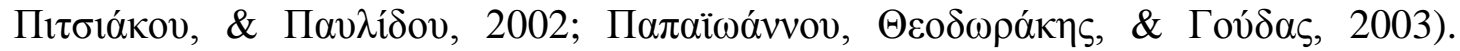

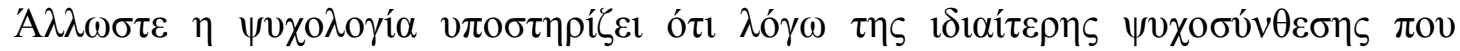

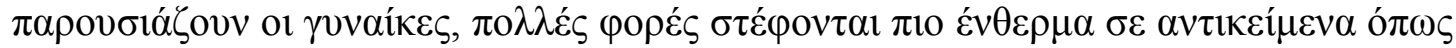

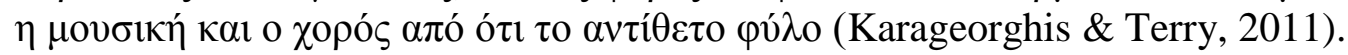

\section{$\Sigma v \mu \pi \varepsilon \rho \alpha ́ \sigma \mu \alpha \tau \alpha$}

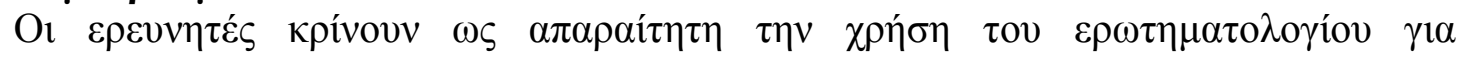

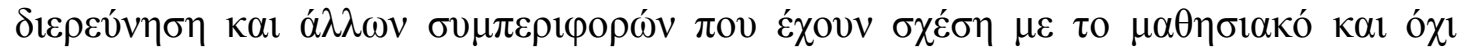

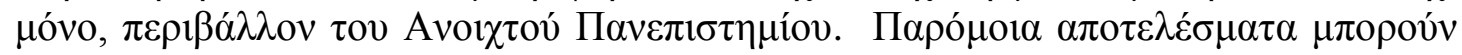

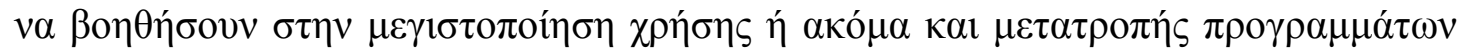

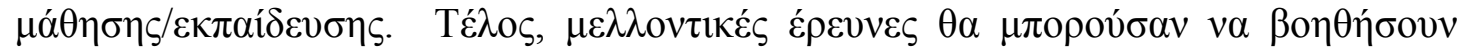

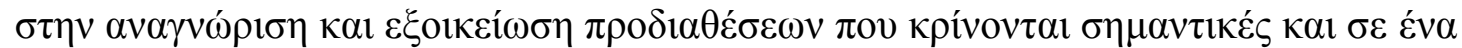

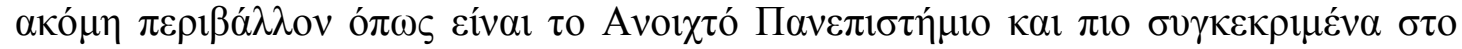

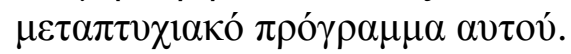

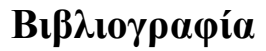

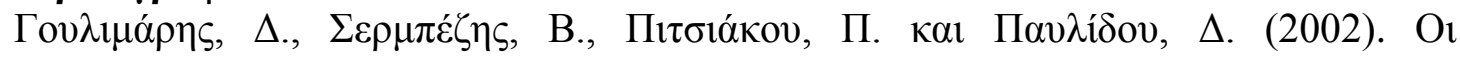

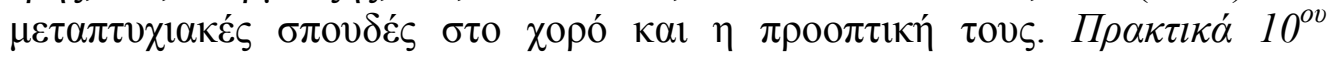

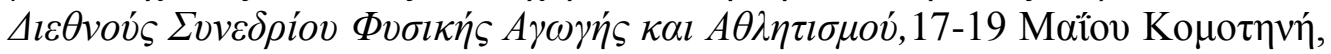
ТЕФАА $\triangle \Pi \Theta, \sigma \varepsilon \lambda .166$

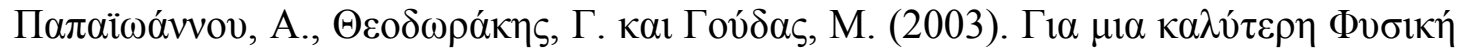

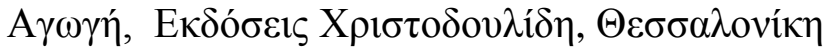

Ajzen, I. (1988). Attitudes, personality and behavior ( ${ }^{\text {st }}$ ed.), Milton Open University Press, UK

Ajzen, I. (1991). The theory of planned behavior. Organizational behavior and human decision processes, 50, pp. 179-211

Ajzen, I. (2005). Attitudes, personality and behavior ( $2^{\text {nd }}$ ed.). Milton Open University Press, UK

Ajzen, I. and Fishbein, M. (1980). Understanding attitudes and predicting social behavior. Englewood Cliffs, NJ, Prentice-Hall

Ajzen, I. and Madden, T.J. (1986). Predictions of goal-directed behavior: Attitudes, intentions and perceived behavioral control. Journal of Experimental Social Psychology, 22, pp. 453-457 
Bebetsos E., Kouli O. and Antoniou P. (2007). Attitudes and Behaviors of University PE Students Towards the Use of Computers. International Journal of Computer Science in Sport, 6 (1), pp. 55-63

Bebetsos E. and Antoniou P. (2008). University students' differences on attitudes towards computer use. Comparison with students' attitudes towards physical activity, Interactive Educational Multimedia, 17, pp. 20-28

Bebetsos E. and Antoniou P. (2009). Gender differences on attitudes, computer use and physical activity among Greek University students. Turkish Online Journal of Educational Technology, 8 (2), pp. 63-67

Karageorghis, C.I. and Terry, P.C. (2011). Inside sport psychology. Champaign, IL: Human Kinetics

Theodorakis, Y. (1994). Planned behavior, attitude strength, self-identity, and the prediction of exercise behavior. The Sport Psychologist, 8, pp. 149-165 